effect on these lower spikelets since those developing higher on the rachis were affected.

Plant 4, which was sprayed at a later stage, demonstrates this point still further, and plant 5 shows the effect of spraying when only the last few spikelet primordia remained to be initiated before the growing point ceased to grow.

Plant 6 was sprayed when all the spikelet primordia were laid down and had progressed beyond the early phase of their initiation.

In every case the spikelet primordia below the point of abnormality have developed quite normally, and it is known that in practice they produce normal fertile flowers and seed. Thus it appears to me that, while Dr. Petersen's work may show translocation to be a contributory factor in the increasing resistance of barley with age to damage by the plant growth regulator herbicides, the age (or more correctly the stage of development) of the leaf or spikelet primordia must still be regarded as a major factor governing susceptibility to damage.

A great deal of work has been carried out here on the effect of the plant growth regulator herbicides on cereals and it has been found that the situation is highly complex. We have found considerable variation to occur in the behaviour of different growth regulators. For example, in barley, $\alpha(2: 4$-dichloro-3methylphenoxy)propionic acid and $\alpha(2: 4: 5$-trichlorophenoxy)propionic acid both cause spikelet abnormality (usually sterility) even when applied at a late stage of primordial development, while others, such as $\alpha$ (4-chloro-2-methylphenoxy)propionic acid, cause relatively little abnormality even when applied at the most sensitive stage. Work is in progress, using carbon-14 labelled growth regulators, to attempt to resolve the causes of these differences. I am indebted to Dr. Petersen for bringing to my notice the important part that translocation may play.

Research Department,

E. L. LEAFE

Agricultural and Horticultural Division,

Boots Pure Drug Co., Ltd.,

Lenton Experimental Station,

Nottingham. Jan. 6.

${ }^{1}$ Nature, 182, 1685 (1958).

\section{'Karathane' : the Value of its Differential Fungicidal Effect in Relation to Mildew and Yellow Rust of Wheat}

EXPERIMENTAL work with yellow rust (Puccinia glumarum (Schm.) Erikss. and Henn.) on wheat seedlings under glasshouse conditions has been considerably hampered by epidemics of cereal mildew (Erysiphe graminis D.C.). Control of these epidemics has proved difficult because environmental conditions in a normal glasshouse cannot be kept within narrow limits and because fungicides which have been effective against the mildew have also been toxic to the rust. Reports of the effectiveness of 'Karathane' (2:4-dinitro-6-(1-methyl heptyl) phenyl orotonate) when used either as a wettable powder or as a fumigant for the control of many powdery mildews but no other disease prompted us to examine the possibility of its being of use in this instance.

A number of seedlings bearing uredosori of yellow rust were fumigated with 'Karathane' when the first mildew pustules appeared. Subsequent development of both diseases was compared with that on similar, untreated seedlings. The flush of mildew pustules, which typically follows 4-5 days after the initial infection, occurred on the untreated seedlings but not on those fumigated. Mycelial growth of the mildew was not entirely suppressed but the pustules were thinner and more widespreading than normal. The yellow rust was apparently unaffected and continued to produce uredosori in a typical zonation.

In subsequent experiments it was found that both rust-resistant and rust-susceptible seedlings fumigated with 'Karathane' 1-10 days before and 2-10 days after inoculation with uredospores gave the anticipated host reaction. Furthermore, uredospores taken from plants only $20 \mathrm{hr}$. after fumigation successfully infected the seedlings to which they were inoculated.

A microscopical examination of both fungi was made $20 \mathrm{hr}$. after fumigation. The yellow rust uredospores from treated and untreated seedlings were indistinguishable, but some differences were noted in the mildew spores. Oidia were stained with cotton blue in lacto-phenol before examination. Those from treated plants had a granular, unevenly stained cytoplasm and the nuclei were well stained and clearly visible. Some bursting and irregularities of the spore wall was also noted. Oidia from untreated plants were more uniformly and more densely stained and consequently the nucleus was not as clearly visible.

During experiments with seedlings it was found that the mildew could be kept under control without any adverse affects on the yellow rust for a period of at least 8 weeks by regular fumigation with 'Karathane' immediately fresh mildew pustules appeared. Under the conditions prevailing during the experimental period, this was at about 10-12 day intervals.

\section{A. Doling \\ SHIRLEY HEPPLE* \\ National Institute of Agricultural Botany, Cambridge. Jan. 5. \\ * Present address: School of Agriculture, University of Cambridge. \\ BIOLOGY \\ Estrous Cycle, Gestation Period and Parturition in the Marsupial Trichosurus vulpecula}

Colonies of the brushtailed possum, Trichosurus vulpecula Kerr., a marsupial, have been kept in Sydney since May 1954 and in Adelaide since January 1957. Breeding seasons were studied, in both places, in the local wild populations from which the captive animals were derived. Breeding in the Sydney colony began in June 1955 and in the Adelaide colony in March 1957. To date 46 specimens (including 16 second-generation young) have been born in captivity. Only one young is normally reared each year in the wild in the Sydney area, but it seems that many wild females in the Adelaide area rear two young each year.

T. vulpecula is polyostrous. Daily vaginal smears were taken from 13 females in the Adelaide colony over periods of up to 10 weeks. In these the cestrous cycle varied in length from 21 to 30 days (average $24 \frac{1}{2}$ days). (Estrus did not occur in lactating females with young in the pouch, but if the pouch-young were removed such females came into oestrus between 4 and 10 days later. Females which lost pouch-young often produced a second young soon afterwards, 